\title{
Antiviral activity of (E)-cinnamaldehyde revisited with nanoscience tools
}

\author{
Arunava Goswami and Ayesha Rahman
}

Biological Sciences Division, Indian Statistical Institute, 203, B. T. Road, Kolkata 700108, West Bengal, India.

Corresponding author: Arunava Goswami; agoswami@isical.ac.in [A. G.]

\begin{abstract}
Contradictory results have been reported regarding the anti-viral activity of (E)-cinnamaldehyde, a major constituent $(\sim 69 \%)$ of cinnamon. Here we show that (E)-cinnamaldehyde alone has very low antiviral property contrary to the belief of commoners. There are early sporadic reports in ancient medicinal practices that fine sand was used for increasing the efficacy of antiviral drugs. Can we increase the efficacy of (E)cinnamaldehyde marginally by using one of the major constituents of sand like silica? Yes, when nanosilica is used as a carrier during (E)cinnamaldehyde administration, the antiviral efficacy of the resultant cocktail increases marginally. Therefore, $(E)$-cinnamaldehyde consumed for centuries in tribal therapy as well as in alternative medicine are largely belief based and does not yield good result till date, when subjected to rigorous scientific investigation.
\end{abstract}

Cinnamon has been consumed by patients suffering from various viral diseases for centuries. Recently, there is an increasing trend among HIV patients towards the use of cinnamon oil as therapy against HIV in the developing and developed nations including India. Urgent scientific investigation is therefore warranted to find out the efficacy level of major constituents of cinnamon oil like (E)-cinnamaldehyde as antiviral antidote.

Gruenwald et al. carefully reviewed the literature on beneficial effect of cinnamon on human health in $2010^{1}$. They reported that only a very small number of well managed case controlled clinical studies have been reported. As a result, it is extremely difficult to make definite conclusions about the potential health benefits of cinnamon. Here we report that we support the conclusions made by Gruenwald et al. We used $100 \%$ lethal silkworm viral disease, Bombyx mori nuclear polyhedrosis virus (BmNPV) as a model system. The mode of action of this double stranded DNA virus is close to HIV and Ebola virus. Due to restriction on using HIV and Ebola in our laboratory, we chose to work on BmNPV. We are confident that our result will be applicable to HIV and Ebola as well.

Fresh stem barks of Cinnamomum zeylaicum were collected. (E)-cinnamaldehyde was prepared using standard protocol ${ }^{2}$. Nanosilica was prepared from Tetra-methoxysilane (TMOS) via wet chemical process and surface functionalized with 3mercaptopropyl-trimethoxysilane (MPTS) as per standard protocol mentioned elsewhere ${ }^{3}$. Nano-silica as well as mixture of nanosilica and (E)-cinnamaldehyde were characterized using DLS, SEM, TEM, UV-spectroscopy, Thermogravimetric Analysis (TGA) and Differential Scanning Calorimetry (DSC) studies.

Polyhedra from B. mori were isolated following standard protocol ${ }^{4}$. One day old age matched $5^{\text {th }}$ instar larvae of $B$. mori were artificially infected following standard protocol ${ }^{5}$. First, normal larvae were inoculated with BmNPV polyhedra via hemoceol 
route. Then, following treatments were given- nanosilica alone, (E)-cinnamaldehyde, nano-silica plus (E)-cinnamaldehyde. For each treatment 200 normal larvae were used along with normal larvae injected with deionized water and BmNPV alone as controls. The percentage of surviving larvae was recorded at different time intervals. Each experiment was tested in triplicate and was repeated five times.

DLS data showed that average particle size (APS) of the nanosilica was $133 \pm 38 \mathrm{~nm}$ (Fig. 1; histogram set 2). Whereas custom made nanosilica purchased from M/S MKnano Inc., Canada (Fig. 1; histogram set 1) and nanosilica-(E)-cinnamaldehyde complex (Fig. 1; histogram set 3) had 40-60 nm APS and 110-130 nm APS respectively.

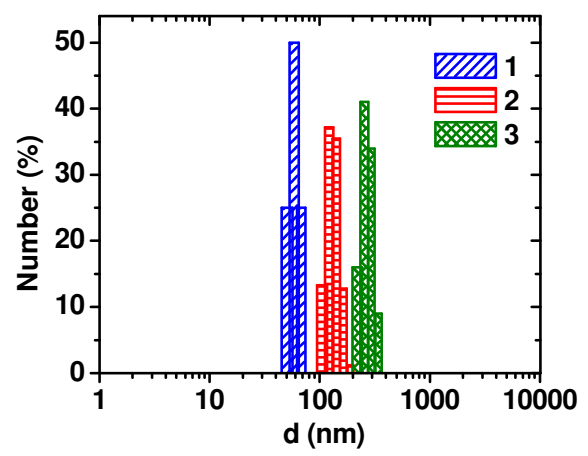

Fig. 1. Hydrodynamic diameter distributions for custom made nanosilica purchased from M/S MKnano Inc., Canada (histogram set 1), nanosilica (histogram set 2) and nanosilica-(E)-cinnamaldehyde complex (histogram set 3 ) measured by DLS.

DLS measures hydrodynamic diameter. Therefore, exact average size of the particles was determined by measuring the size of around 200 particles in the Scanning electron microscopy (SEM) and Transmission electron microscopy (TEM) photographs (Fig. 2). Similar data were obtained for custom made nanosilica and nanosilica-(E)-cinnamaldehyde complex (data not shown).

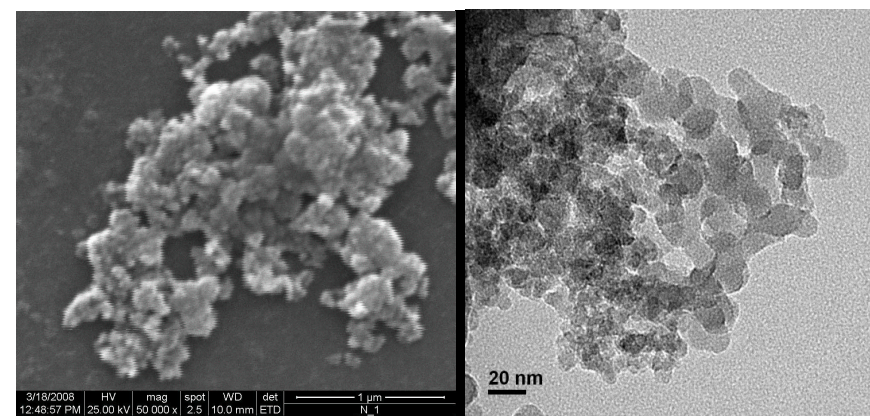

(a)

(b)

Fig. 2. (a) SEM and (b) TEM micrographs of nanosilica.

UV-VIS spectroscopy studies were done to find out whether any chemical changes occured in (E)-cinnamaldehyde when it binds with nanosilica. Fig. 3 (graph 1) shows 
the absorbance spectra of (E)-cinnamaldehyde alone with a characteristic peak at 285 $\mathrm{nm}$. The nanosilica-(E)-cinnamaldehyde complex showed peak at the same positon (Fig. 3. graph 2) suggesting that the chemical properties of (E)-cinnamaldehyde did not change in the nanosilica-(E)-cinnamaldehyde complex. As expected, nanosilica alone did not show any considerable absorbance and the relative change of absorbance is also low (Fig. 3. graph 3)

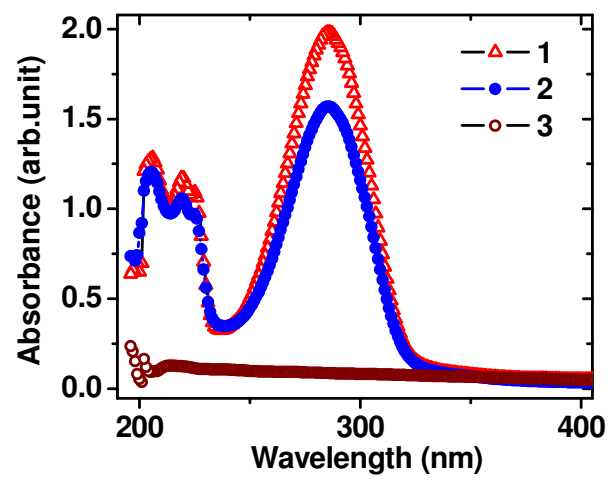

Fig. 3. UV-VIS absorbance spectra of (E)-cinnamaldehyde (graph 1), nanosilica-(E)cinnamaldehyde complex (graph 2) and nanosilica alone (graph 3)

TGA analysis [Fig. 4, panel (a)] showed that major weight loss of nanosilica occurs within $150^{\circ} \mathrm{C}$ due to the loss of the functional coating of the nanosilica. Considerable weight loss of nanosilica-(E)-cinnamaldehyde complex was observed above $250^{\circ} \mathrm{C}$ suggesting that weight loss above $150^{\circ} \mathrm{C}$ is primarily due to the decomposition and evaporation of (E)-cinnamaldehyde attached with nanosilica. Similarly endothermic dip in DSC signal of nanosilica [Fig. 4, panel (b)] is observed at $95^{\circ} \mathrm{C}$ which can be assigned to desorption of the organic coating of the nanosilica. In case of nanosilica(E)-cinnamaldehyde complex similar endothermic peak is observed at $67^{\circ} \mathrm{C}$ which indicates desorption of (E)-cinnamaldehyde from nanosilica. An exothermic peak at $190^{\circ} \mathrm{C}$ is observed in nanosilica-(E)-cinnamaldehyde complex which is probably due to the decomposition of (E)-cinnamaldehyde. These results all together support that when mixed together, nanosilica binds with (E)-cinnamaldehyde to form the nanosilica-(E)-cinnamaldehyde complex.

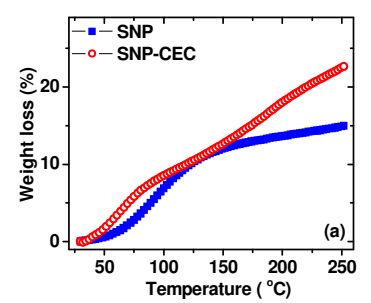

(a)

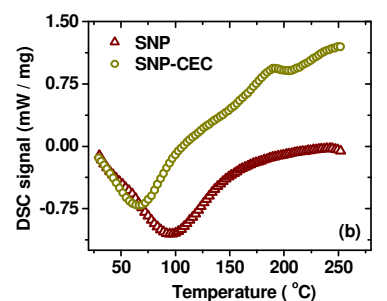

(b)

Fig. 4. (a) Thermo gravimetric curves of nanosilica and nanosilica-(E)cinnamaldehyde complex and (b) Differential Scanning Colorimetry thermograms of nanosilica (designated as SNP) and nanosilica-(E)-cinnamaldehyde complex (designated as SNP-CEC). 


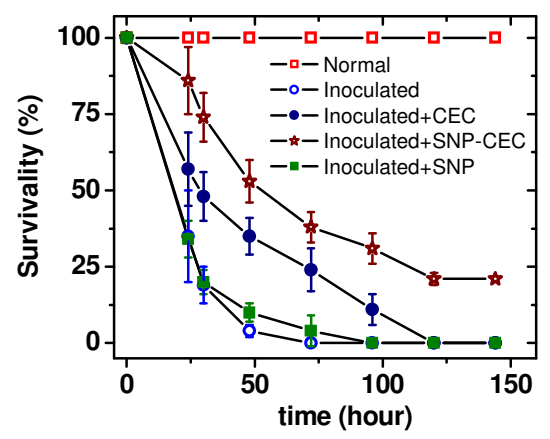

Fig. 5. Normal: Healthy larvae injected with deionized water; Innoculated: Larvae injected with BmNPV alone; Innoculated+CEC: BmNPV infected larvae treated with (E)-cinnamaldehyde alone; Innoculated+SNP-CEC: BmNPV infected larvae treated with nanosilica-(E)-cinnamaldehyde complex; Innoculated+SNP: BmNPV infected larvae treated with nanosilica alone.

Fig. 5 showed that $100 \%$ larvae injected with BmNPV inocula or nanosilica alone died within 96 hours post infection. 11\% survival was noted in BmNPV infected larvae treated with (E)-cinnamaldehyde alone after 96 hours. More than $25 \%$ infected larvae completed their lifecycle when treated with nanosilica-(E)-cinnamaldehyde complex. This result clearly shows that there is a marginal improvement of antiviral activity of (E)-cinnamaldehyde when it is administered along with nanosilica.

\section{Acknowledgements}

This work was supported by the Department of Biotechnology (DBT), New Delhi, grants (BT/PR9050/NNT/28/21/2007 and BT/PR8931/NNT/28/07/2007 to AG) and NAIP-ICAR-World Bank grant (Comp-4/C3004/2008-09; Project leader: AG) and ISI plan projects for 2001-2011 to A. G. Authors wish to thank Dr. B. Barik for providing authentic cinnamon bark from the collection of Central Research Institute (Ayurveda), India.

\section{References}

1. Gruenwald, J., Freder, J. \& Armbruester, N. Cinnamon and health. Crit. Rev. Food Sci. Nutr. 50, 822-834 (2010)

2. Unlu, M., Ergene. E., Unlu. G. V., Zeytinoglu, H. S. \& Vural, N. Composition, antimicrobial activity and in vitro cytotoxicity of essential oil from Cinnamomum zeylanicum Blume (Lauraceae). Food Chem. Toxicol. 48, 3274-3280. Epub 2010 Sep 7 (2010)

3. Stober, W. \& Fink, A. Controlled growth of mono-dispersed silica spheres in the micron size range. J. Colloid. Interface Sci. 26, 62-69 (1968)

4. Rahman, M. M. \& Gopinathan, K. P. Systemic and in vitro infection process of Bombyx mori nucleopolyhedrovirus. Virus Res. 101, 109-118 (2004)

5. Keddie, B. A., Aponte, G. W. \& Volkman, L. E. The pathway of infection of Autographa californica nuclear polyhedrosis virus in an insect host. Science 243, $1728-1730$ (1989) 